\title{
Epidemiological Surveys Embedded in Technical Assistance to Tuberculosis Control Programmes
}

\author{
Frank van Leth ${ }^{*}, 1$ and Eveline Klinkenberg ${ }^{1,2}$ \\ ${ }^{I}$ Department of Global Health, Academic Medical Center, University of Amsterdam, Amsterdam Institute for Global \\ Health and Development, The Netherlands
}

${ }^{2}$ KNCV Tuberculosis Foundation, The Hague, The Netherlands

\begin{abstract}
Many National Tuberculosis Programmes (NTPs) receive Technical Assistance (TA) for the implementation and evaluation of their TB-control strategies. KNCV tuberculosis Foundation (KNCV) in the Netherlands is a TA organization providing assistance for many years in over 30 countries.

The NTPs use their own surveillance data to assess TB control strategies. These data are not accurate enough to serve as valid epidemiological parameters. $\mathrm{KNCV}$ adds an epidemiological research component to the general TA. Epidemiological research does give the evidence-base needed for TB control. Embedding this research in continued programme support from TA consultants has been fruitful.

This paper details KNCV's experiences with such TA plus epidemiological research approach, specially in conducting large-scale population-based surveys: tuberculin surveys, TB prevalence surveys, and drug resistance surveys. The lessons learned include the added value of long-term programme support, of a thorough knowledge of the survey's situational context, of in-country capacity building, and of guidelines for guiding, as opposed to dictating, research.
\end{abstract}

Keywords: Tuberculosis, tuberculin, drug resistance, prevalence, surveys, technical assistance, epidemiology, KNCV.

\section{BACKGROUND}

National Tuberculosis Programmes (NTPs) use their routinely collected data, such as the number of patients identified in a given year, to measure the impact of their tuberculosis (TB) control strategies. These routine surveillance data often lack the completeness and accuracy to serve as valid epidemiological parameters in impact assessments, for which it is essential to have data collected in an unbiased manner. Well-designed population-based surveys are a valuable tool in obtaining these data. However, the design, implementation, and analysis of such surveys demand skills that are often lacking at the required level in resource-poor countries. Research organizations have these skills, but are often not connected to the NTPs, thereby overlooking valuable cues of the context in which these surveys take place. By contrast, the Technical Assistance (TA) consultants who offer programme support to NTPs, are strongly embedded in the context of the NTP, but often do not have the appropriate research skills to advise the NTPs on the conduct of large-scale epidemiological surveys.

KNCV Tuberculosis Foundation (KNCV) has provided TA to NTPs for many years, mainly in Asia and Africa. In the late 1990s, KNCV set up a dedicated research unit to complement the work of the general TA consultants. The

*Address correspondence to this author at the Department of Global Health, Academic Medical Center, University of Amsterdam, Amsterdam Institute for Global Health and Development, Pietersbergweg 17, 1105BM Amsterdam, The Netherlands; Tel: +31 20566 1593;

E-mail: f.vanleth@aighd.org aim was to gather representative and valid data on the tuberculosis burden of the assisted countries, thus providing the evidence-base for TB control. The unit's experienced epidemiologists focused not only on epidemiological research, but on operational research as well. The close collaboration between TA consultants and epidemiologists turned out to have many advantages: enhanced mutual understanding between consultants and researchers, a deepened knowledge of local circumstances and needs in the countries assisted, and a uniform representation to the NTPs and international TB stakeholders. That way, the KNCV's TA set-up made its impact felt beyond the direct NTP support for which the TA was originally devised.

KNCV provides TA to NTPs on a short-term and on a long-term basis. Short-term TA is offered when specific expertise is required for a well-defined problem. However, KNCV's preferential approach to providing TA is to see it embedded in a long-term working relationship with NTPs. Such a relationship facilitates the implementation and evaluation of issues that, by their nature, take a long time to address, like programme planning and building or facilitating local capacity.

The emphasis of the TA provided by KNCV epidemiologists lies with the conduct of population-based surveys in the assisted countries, in order to assess their TB epidemiology. It is precisely through such research that NTP self-assessments can be improved upon and NTP programme execution readjusted. The current manuscript will exemplify KNCV's experience in conducting tuberculin surveys, TBprevalence surveys, and drug resistance surveys. The 
reader's attention is drawn to the fact that long-term KNCV TA for NTPs is provided in Kenya, Tanzania and Vietnam; all other instances of TA by KNCV epidemiologists are on a short-term contract basis in countries other than the three aforementioned.

\section{TUBERCULIN SURVEYS}

In tuberculin surveys, participants receive an intradermal injection with a standard dose of a suspension containing tuberculosis antigens. If the injection elicits an immunological skin reaction, the size of the skin reaction is measured and reported as the test result. These results can be used to calculate a population's TB force of infection - the "Annual Risk of Tuberculous Infection" (ARTI). To arrive at this indicator, large scale tuberculin surveys are done among children (5-14 years). Given their age, a skin induration in children may be used as a proxy for Mycobacterium tuberculosis transmissions within the population that are recent.

Tuberculin surveys are notorious for their methodological challenges [1]. For many years, KNCV has provided training that covers all stages of the process of tuberculin surveys: from protocol development, to proper sampling of populations, the adequate application and interpretation of tests, and the final data analysis and dissemination of results. In Kenya, Tanzania, Vietnam and Nepal, KNCV has assisted NTPs to conduct nation-wide surveys for the past ten to fifteen years. The experiences were used in writing new international guidelines for future surveys [2], with the objective to standardize the performance and interpretation of tuberculin surveys and to make results comparable between different surveys. The main challenges in tuberculin surveys are (i) reading of indurations, (ii) maintaining high standards of field work, and (iii) data analysis.

In order to ensure proper reading of test results, $\mathrm{KNCV}$ uses a three weeks full-time curriculum of training in all aspects and phases of tuberculin surveys. There is a strong emphasis on practical matters, such as administering the suspension and reading the test. The training concludes by performing actual field activities in a pilot setting.

Even after successful pilots, however, there can be systematic deviations from Standard Operating Procedures (SOPs) in the actual study, specially when test readers have become overly confident in their skills. It is only through regular monitoring that such deviations can be identified. If present, there is a need for re-training or, ultimately, replacement of survey staff.

Analysis of tuberculin survey data can be complex and is often beyond the capabilities of the NTP staff. The conventional approach, in which all children with a skin reaction above a certain size are assumed to be infected, is, although often used, inappropriate. $\mathrm{KNCV}$ provides on-thejob training of NTP staff to accommodate TA needs pertaining to data analysis. Consistent TA in the three areas of test reading, standardized field activities, and data analysis has been instrumental in completing numerous tuberculin surveys.

In Tanzania, tuberculin surveys were conducted in 5-year cycles between 1983 and 2003. The four surveys showed a declining trend in ARTI over the 20-year period, independently of the analytical approach used in identifying the children [3]. The decline that was observed was nationwide, i.e. without marked exceptions in districts, and cooccurred with an ever growing number of notified TB patients, due to the emerging HIV-epidemic. The authors concluded that these results reflected adequate case finding and treatment of TB patients, with consequent curbing of active transmission, and that HIV-infection did not affect TB transmission at the population level.

Kenya conducted three national tuberculin surveys between 1986 and 2006. Here, the findings contrasted with those in Tanzania. First, the decline in ARTI showed differential changes between districts $[4,5]$. Second, the two surveys of 1986 and 1996 showed a strong effect of HIV on TB transmission. The 2006 survey showed no decline in ARTI. This led to the conclusion that TB control activities needed to be improved throughout the country to reduce transmission [6].

Six selected districts in Vietnam carried out three rounds of tuberculin surveys between 1990 and 2000. The data obtained were used to assess if the introduction and scalingup of the Directly Observed Therapy, Short course strategy (DOTs) had made any impact. But relating changes in ARTI to changes in age-standardized notification rates showed that despite five years of nation-wide implementation of DOTs there was no clear reduction in TB transmission [7]. The same data were used at a later stage to show that the increased notifications of TB in young males were more likely to be associated with increased risk of disease progression than with transmission [8].

TA support in Nepal was not embedded in long-term programme support with repeat surveys, but was offered as a one-off activity with a single survey. There were ARTI differences between different ecological zones in the country. In the absence of adequate data to put the findings into a proper context, the authors concluded that a repeat survey was needed within a time-frame of five to seven years, in order to assess the impact of national TB control activities [9]. Without an on-going collaboration with the NTP, KNCV was unable to facilitate the implementation of this recommendation. To this date, no information on (the intention of) such a repeat survey is available.

The single survey estimate in Nepal raises questions about the usefulness of tuberculin surveys in view of their complex design, potentially biased analyses, and high costs. Without a way of putting findings into the context of previous surveys or of other epidemiological data, a single estimate is not very informative. One epidemiological marker that is widely used as an indicator for progress in TB control is the case detection rate (CDR). In a pivotal paper, Styblo argued that ARTI calculations could be related mathematically to smear-positive TB incidence, and from there to the CDR [10]. According to Styblo, the relationship between the two measurements was mathematically fixed: each per cent point of ARTI was associated with fifty new smear-positive patients per 100.000 per population. Styblo based his relationship on mortality and prevalence data from the 1950s and 1960s. Van Leth et al. showed that such a mathematical proportion did no longer hold in times of effective and large-scale TB treatment, and proposed to drop 
the 'Styblo rule' as a widely used epidemiological marker [11].

\section{TB PREVALENCE SURVEYS}

TB prevalence surveys assess a population's burden of active TB disease. Whereas a single tuberculin survey is not informative (see above), a single TB prevalence survey is, since there are methods for relating prevalence of disease to the CDR. Prevalence and incidence are related in the duration of the disease. Alternatively, the Patient Diagnostic Rate measures the rate at which prevalent TB cases are detected by the NTP, and is the ratio between the number of notified cases and the prevalence of the disease. From here, the CDR can be estimated [12]. Hence, a single TB prevalence survey can estimate the impact of TB control activities provided by NTPs. The underlying methodology still holds good today, as opposed to the 'Styblo rule.'

Interest in $\mathrm{TB}$ prevalence surveys has increased substantially over the last ten years. Before that, they were mainly conducted in Asian countries with the support of the Japanese Anti-Tuberculosis Association (JATA). Expertise with such surveys in sub-Saharan Africa was lacking. One major impetus to engage in such surveys are the United Nations' Millennium Development Goals (MDGs). The sixth goal proposes to have halted by 2015 and begun to reverse the incidence of major diseases, among them tuberculosis [13]. With 2015 approaching, a few Asian countries will be able to conduct multiple surveys to assess this target, while most of the others will need all their efforts in completing a single survey in time.

The WHO Global Task Force on TB Impact Measurement, from June 2006 [14], was set up in order to measure progress towards MDG goal six. One of the main activities is to assist countries in the design and implementation of TB prevalence surveys. Epidemiologists from $\mathrm{KNCV}$ joined this Task Force due to their unique position: they were already providing TA to NTPs in subSaharan African countries and had already started to assist these countries in designing and implementing TB prevalence surveys [15].

One of the first activities of the Task Force was to organize workshops for countries planning a TB prevalence survey. WHO and TA organizations such as KNCV, JATA, and Center for Disease Control and Prevention closely cooperate within the Task Force, and even more so in the workshops. The blueprints for the initial design of countryspecific protocols were KNCV study protocols deriving from TA given in Vietnam and Tanzania. All experts reworked their experiences into a first version of written guidelines for TB prevalence surveys. After the successful implementation of the first surveys, the guidelines were updated and expanded into a second version that provided more detailed guidance [16]. This book is still at the heart of TB prevalence survey design and implementation. Its detailed guidance enables countries to engage more in all parts of the design of survey activities. At the same time, they assure the proper level of standardization.

KNCV epidemiologists provide TA for the national TB prevalence surveys in Eritrea, Vietnam, Bangladesh, Pakistan, Tanzania, and Rwanda. They have also supported the development of protocols and SOPs in countries that are still preparing to start field data collection, such as Ghana, Kenya, Zambia, and Botswana. The main challenges encountered here are: (i) managing long preparation time, (ii) choice of design, (iii) monitoring activities, and (iv) data management.

Between initial planning and actual implementation of TB prevalence surveys several years may elapse. There are many reasons for this: difficulties with procurement, with obtaining adequate funding, with long ethical review processes, and with inadequate human resources in the NTP. When TA is embedded in long-term support, survey preparation is more easily kept on the agenda of the stakeholders, even in times of slow progress. In situations with no such long-term collaboration, delays in progress can lead to interruptions of the TA provided, due to expiring contracts and funding.

Initially, the Task Force geared the design of TB prevalence surveys to country-specific resources and funding. A crucial variable in designs was the inclusion or exclusion of a screening step using X-ray. In Eritrea, KNCV provided TA without X-ray but with microscopic examination of sputum specimens for all survey participants. This turned out to be feasible and affordable; and it yielded valid data on smear-positive tuberculosis in the population [17]. In Bangladesh, where KNCV had been invited to provide TA, the same design was used. A methodology without X-ray screening simplifies the implementation of the survey. A major drawback, however, is that only smearpositive patients can be detected. In settings with an established HIV-epidemic - and this includes most subSaharan African countries - this would potentially lead to a biased estimate of the TB burden. After intense deliberation, the members of the Task Force decided on a single survey design with X-ray screening as the new standard.

What experience in TA provision cannot fail to hammer home, is that no two countries are alike. This means that even with highly standardized guidelines from the Task Force, survey activities will always be adapted to local circumstances. But adaptations may slide into deviations, and where protocols become loose, data collections become biased. It is therefore imperative to have frequent monitoring visits. KNCV found this out in Pakistan. The survey took place during a difficult time, in a fragile safety situation. Survey supervisors and TA consultants were prevented from visiting survey sites for monitoring purposes due to security threats. Consequently, deviations from the survey protocol and the SOPs could not be identified, data entry was delayed, and problems in data collection and recording had to be corrected as much as possible at the analysis stage, since they had not been corrected in the field.

One of the main challenges in any of the current surveys is data management and analysis. This seems to be the most context-dependent aspect of TB prevalence surveys. In the design phase, the survey guidelines suggest how to go about organizing data management in the field and at the central level, but survey coordinators cannot yet draw on the experience needed to envision what it entails. Most NTPs, for their part, simply cannot deal with sample sizes reaching 45.000-65.000, with clustered field activities taking place in just one week, and the inability to correct data collection after the study team has left the cluster. Still, data collection 
needs to be reliable. KNCV epidemiologist aim for simultaneous data entry and data collection in order to optimize data management. This requires adequate human resources and budget.

Often, NTPs only request assistance with the primary analysis of the prevalence of disease, and this can easily be provided within the context of TA. It is the analysis of additional data that has often not been factored in. KNCV has good experiences with building local research capacity by incorporating all additional analyses into $\mathrm{PhD}$ programmes. This brings together a designated local researcher and a TA consultant, ensures a timely analysis within the time-frame of the $\mathrm{PhD}$-programme, and makes sure that all data collected in the survey are used for policy development.

TB prevalence surveys are expensive. It is estimated that in Asia the inclusion of a single participant will cost between $\$ 14$ and $\$ 29$, depending on the exclusion or inclusion of an X-ray component. For Africa, the estimates are between $\$ 25$ and $\$ 67$ [16]. With sample sizes between 45.000 and 65.000 this amounts to $\$ 630.000$ (no X-ray) and $\$ 1.2$ million (with $\mathrm{X}$-ray) in Asia, and between $\$ 1.2$ million and $\$ 4.3$ million in Africa. One way of making the most of the money spent is to design add-on studies that can piggyback on the survey's data collection. There are add-on studies in all surveys for which KNCV has provided TA, but there are two limiting factors to add-ons. First, add-on studies affect field activities and this may have an undesirable impact on the survey's primary objectives. Second, add-on studies should fit the sampling strategy used for the survey, in order to avoid biased designs [18]. Nevertheless, if well designed, add-on studies can provide unique information, not only regarding contents (TB epidemiology), but also regarding conduct (surveying as such). Such information is valuable to NTPs and policy makers.

In Vietnam, KNCV has an add-on study on healthseeking behaviour by means of an in-depth interview. It was found that half of the participants with a persistent cough (which is an indicator for clinical work-up to TB) visited a health-worker. This health-worker, however, was mainly based within pharmacies where no diagnostic procedures were initiated [19]. This add-on thus tells us at which locations TB diagnostic procedures can be strengthened. Inclusion of these health-seeking behavior data has now become a standard recommendation in the second edition of the survey guidelines; it has also been added as a secondary objective of TB prevalence studies.

In Vietnam and Bangladesh, information on household assets is used as a proxy for socio-economic position (SEP) [20]. In Vietnam, this add-on is an extension of earlier work by the World Bank. Rapid assessment of SEP is formulated in terms of a list of nine assets that reliably predict a household's monetary expenditure [21]. In both surveys, KNCV found a strong relationship between having TB and having a low SEP. In Bangladesh, information on SEP made it clear that the NTP did not reach the poorest parts of the community where TB prevalence was highest [22]. This sparked a debate within the NTP on how to improve the coverage of NTP activities.
In Vietnam, an add-on study assesses the diagnostic value of screening for the presence of a persistent cough $(>2$ weeks). Persistent coughing is a strong predictor for TB in a clinical setting, but the study showed that this can not be translated to population-based screening. Additional chest Xray screening in the survey setting increases case-finding by $62 \%$ [23]. In Bangladesh, a similar conclusion was drawn from an add-on study. The routine clinical approach is to macroscopically judge the quality of submitted specimens for microbiological examination. But this approach fails in a survey setting. The add-on, using microscopic parameters, showed that over $50 \%$ of the specimens macroscopically judged as being saliva, and hence inadequate for further assessment, were in fact proper sputum samples [24].

In Rwanda and Tanzania, current KNCV add-on studies are likely to yield information that cannot be gleaned from any other survey. In Rwanda, there are participants who were eligible for sputum examination, smear negative in the field laboratory, but culture positive in the central laboratory. Here, the add-on traces the symptomatology of these participants from their inclusion in the survey onwards. The information can play a role in TB modeling studies on diagnostic algorithms. In Tanzania, a random sample of the population is subjected to all survey activities, including sputum examination. This is unique. It will provide an opportunity to assess (i) population-attributable risk factors for $\mathrm{TB}$, and (ii) the extent to which screening algorithms contain a 'verification bias,' which occurs when only screening-positive participants are examined further for TB [25].

The results of TB prevalence surveys differ widely from yearly provided WHO estimates. The survey in Eritrea estimated a prevalence of smear-positive TB that was more than $50 \%$ lower than the WHO estimate of that time [17]. The same was seen in the survey in Bangladesh [26]. As mentioned, the TB prevalence surveys in Eritrea and Bangladesh excluded chest X-ray as a screening step. Initially, this exclusion of the X-ray screening step was seen as the cause of the markedly lower estimates in these surveys as compared to the WHO estimates. However, the recent survey in Ethiopia, which did have chest X-ray included as a screening step, also reported a markedly lower prevalence than estimated by WHO (unpublished data). This casts doubt on the assumption that there is any simple association between X-ray inclusion and convergence between prevalence estimates from the surveys and those of WHO. The survey in Vietnam could be seen to underline this argument. Here, X-ray inclusion in the survey led to findings that were still not in line with WHO estimates, but this time it was the survey estimate that was markedly higher than the WHO estimate [27].

The existence of the differences between the estimates of TB-prevalence surveys and WHO calls for elucidation rather than polarization. Data are the results of operations, and where these operations differ, the resulting data will differ. WHO estimates are based on interpretation and mathematical modelling of routine data provided by the NTPs on a yearly basis and therefore rely on passive case detection. By contrast, TB prevalence surveys provide data from active case detection through direct measurement in samples of the general population. When the operations leading to the data 
have been defined clearly this makes them, in principle at least, repeatable and controllable. As such, each data source gives additional information which should be weighed to arrive at the best possible evidence-base. TA consultants play an important role in facilitating this process. Especially when the TA is embedded in long-term programme support, the consultants' strategic position in both the local environment of the NTP and such international groups can be used to maximum advantage.

\section{DRUG RESISTANCE SURVEYS}

Current TB control is seriously hampered by both the HIV epidemic and the emergence of drug resistance [28]. WHO has issued international guidelines on the conduct of drug resistance surveys (DRS) because of its importance: knowing the extent to which there is drug resistance to TB medication helps to design interventions that curb further spreading of resistance [29]. As with tuberculin surveys and TB prevalence surveys, drug resistance surveys need careful attention. KNCV assists several countries with national or regional DRS, amongst others Vietnam, Tanzania, Zimbabwe, and China. The main challenges encountered are (i) obtaining an adequate sampling frame, (ii) organizing timely transportation of sputum specimens, and (iii) ensuring proper data management.

The typical sampling frame in a DRS is defined in terms of smear-positive tuberculosis at each TB diagnostic facility in the country or region. For sampling to be valid, the frame needs to consist exclusively of such participants. If not, the frame is void, and random sampling becomes impossible no matter how well the DRS is carried out otherwise. TA will therefore focus on helping NTP staff to use their surveillance data to compile an appropriate sampling frame. In countries that are unable to provide such data, a presurvey assessment of the facilities is needed, and this may take a considerable amount of time.

Smear microscopy relies on the availability of sputum specimens from participants at the research laboratory. This entails logistics: the specimens need to be collected, stored and transported in controlled conditions (e.g. cooling), and have to arrive at a laboratory within three-four days after collection. TA here is unthinkable without knowledge of local infrastructure. In Tanzania, pre-paid arrangements with bus companies ensured timely transportation of specimens, with SMS texting to monitor the dispatch of the specimens. That way, mean transportation time was well within the crucial time-window, even from remote clusters in the survey [30]. These DRS logistics later became the template for Tanzania's TB prevalence survey, without ever having been intentionally defined as a test-case for it. It was a spinoff from long-term TA provision and might not have presented itself within short-term contract assistance.

Laboratory activities for a DRS do not markedly differ from routine procedures. Laboratories that have shown themselves capable of sputum culture and drug sensitivity testing can be included in the survey. What does differ between routine setting and survey setting is data recording and data management. It is here, then, that TA comes in with standardization and regular monitoring to ensure a one-toone transfer of data from one setting to the next. In Tanzania, for instance, data distortion did occur due to a lack of a similar lay-out in data capture and recording. The order of the columns for specific drugs did not match that of the routine register, causing serious data entry errors. This distortion could only be detected and corrected with the strictest of management procedures, and took up valuable time. As with the TB prevalence survey design, data management is often neglected in DRS design. TA needs to budget for data management and other practical operations if epidemiology is to succeed in making helpful contributions to NTPs.

International guidelines notwithstanding, there will always be a need to adapt procedures to local circumstances. In Tanzania, even bottlenecks well-known from any field study ever done proved to be challenging despite careful planning. This ranged from the episodic (leaking specimen tubes) and the habitual (poor recording) to the systematic (delayed data management). Implementation will never be without hurdles [31]. But this, in itself, highlights the need for continued and systematic TA to the survey teams.

As with TB prevalence surveys, a DRS can be accompanied by add-on studies. In Tanzania, an alternate way of storing sputum specimens (in ethanol) was tried to enable longer transportation times to laboratories. The addon showed that ethanol storing was possible, and that genotypic resistance testing of these specimens provided near-identical results to standard phenotypic resistance testing of specimens stored under default conditions [32]. This finding could have a major impact on future surveys [33].

There are also instances of useful secondary analysis that have not been formally set up as add-on studies. In Vietnam, a survey showed a marked prevalence of streptomycin resistance in younger patients, indicating recent transmission of resistant strains [34]. One of the hypotheses put forward was a relationship between streptomycin resistance and transmission fitness of the Beijing type (prevalent in Vietnam). Newly designed studies with adequate follow-up and molecular typing techniques confirmed this relationship [35]. The surveys in Vietnam and Tanzania made the data available to an international forum to assess the validity of new sampling techniques for a DRS. Lot Quality Assurance Sampling is an efficient way of rapidly assessing whether drug resistance levels are above a pre-defined set level [36].

\section{TA VERSUS IN-COUNTRY CAPACITY BUILDING}

It is has been our intention to describe the extent and impact of KNCV's TA to population-based surveys. KNCV emphasizes local capacity building, ranging from on-the-spot training to full international $\mathrm{PhD}$ programmes. The emergence of local research institutes in low-resource countries is a very positive development; so too is SouthSouth collaboration in research projects. KNCV believes that it is important for TA providers to stimulate such developments. High quality national research and training capacities cannot be established overnight.

\section{LESSONS LEARNED}

KNCV's longstanding TA for epidemiological research has grown into a vast reservoir of operational experience and scientific evidence to draw on in future surveys in other countries. 
Several points have already been made in passing. The most obvious one is that research TA embedded in long-term programme support has some clear advantages over TA provided on a one-off contract basis. The survey's phases of designing, implementing, and analysing each require care. The earlier TB prevalence surveys took several years of designing. Their actual data collection phase ran anywhere between nine and fifteen months. These processes are slow, slower than most contract expiry dates will allow for. But slow does not mean halted, so that even in times of little visible progress, the survey remains at the forefront of everyone's attention. Moreover, rushing into research has invariably led to inferior data collection; and some data simply cannot be corrected at the analysis stage of the survey.

Another point is that long-term TA by an experienced epidemiologist provides opportunities for engaging the national stakeholders. This means that the research request originates from within the TB control programme itself, and that the stakeholders will support its results. This, in turn, ensures that the results will find their way into policy and decision making for future activities. To be able to fulfill this role, the epidemiologist needs more than a mere working knowledge of the survey's couleur locale. Situational parameters such as customs, infrastructure, research capacity, and so on, are vital. They will inform the research questions pertinent to the programme, the design of appropriate sampling schemes, and optimize the use of local research facilities needed to implement the protocols.

Epidemiological surveys are impossible without optimal use of local research capacity. KNCV prefers its research projects to contain capacity building strategies. When the training extends beyond the sphere of immediate results or project-specific activities, that expertise can be used for future projects. An NTP staff capable of collaborating on designing and implementing operational research is an asset to any disease control programme. But access to local research staff does not replace the need for frequent monitoring by the epidemiologist.

The results of epidemiological research need to be made available to the international research community, instead of remaining confined to the national stakeholders and participants. Only then will the results of these, often expensive, activities have their full impact. Estimates on the prevalence of TB disease and drug resistance have found their way into official national and international statistics. As such, they serve as a benchmark for evaluating the effect of future control strategies. KNCV supports this international dissemination by means of assisting in the preparation of reports and of scientific publications.

Arguably the biggest lesson learned from the support given to all these national surveys is that each situation is unique. Standardization is not a lofty ideal, but a necessity; and yet, research needs to adapt itself to local situations. This requires continued reflection within a group of experts in order to identify appropriate solutions to common problems, and, on occasion, to provide innovative solutions to unique problems. Therefore, it is important that guidelines are there to guide, not to dictate. TA providing organizations, such as $\mathrm{KNCV}$, are in a good position to meet such challenges. As such, they are an important asset to international research within the field of TB control.

ABBREVIATIONS
CDR $=$ Case Detection Rate
DOTs $=$ Directly Observed Treatment, Short course
DRS $=$ Drug Resistance Survey
HIV $=$ Human Immunodeficiency Virus
MDG $=$ Millennium Development Goals
NTP $=$ National Tuberculosis Programme
SEP $=$ Socio-Economic Position
TA $=$ Technical Assistance
TB $=$ Tuberculosis
WHO $=$ World Health Organization

\section{CONFLICT OF INTEREST}

Frank van Leth was a full-time employee of KNCV Tuberculosis Foundation from 2005 to 2010; in 2011he was seconded to the organization. Eveline Klinkenberg has been a full-time employee of KNCV Tuberculosis Foundation since September 2007.

\section{ACKNOWLEDGEMENTS}

The authors thank Arjan de Zeeuw for the critical review of the manuscript

\section{REFERENCES}

[1] Rieder HL. Methodological issues in the estimation of the tuberculosis problem from tuberculin surveys. Tuber Lung Dis 1995; 76: 114-21.

[2] Rieder HL, Chadha VK, Nagelkerke NJD, Van Leth F, Van der Werf MJ. Guidelines for conducting tuberculin skin test surveys in high-prevalence countries. Int J Tuberc Lung Dis 2011; 15 Suppl 1: S1-25.

[3] Egwaga SM, Cobelens FG, Muwinge H, Verhage C, Kalisvaart N, Borgdorff MW. The impact of the HIV epidemic on tuberculosis transmission in Tanzania. AIDS 2006; 20: 915-21.

[4] Bosman MC, Swai OB, Kwamanga DO, Agwanda R, Idukitta G, Misljenovic O. National tuberculin survey of Kenya, 1986-1990. Int J Tuberc Lung Dis 1998; 2: 272-80.

[5] Odhiambo JA, Borgdorff MW, Kiambih FM, et al. Tuberculosis and the HIV epidemic: increasing annual risk of tuberculous infection in Kenya, 1986-1996. Am J Public Health 1999; 89: $1078-82$.

[6] Kwamanga D, Chakaya J, Sitienei J, Kalisvaart N, L'herminez R, Van der Werf MJ. Tuberculosis transmission in Kenya: results of the third National Tuberculin Survey. Int J Tuberc Lung Dis 2010; 14: 695-700.

[7] Huong NT, Duong BD, Co NV, et al. Tuberculosis epidemiology in six provinces of Vietnam after the introduction of the DOTS strategy. Int J Tuberc Lung Dis 2006; 10: 963-9.

[8] Buu TN, Quy HT, Qui NC, Lan NTN, Sy DN, Cobelens FGJ. Decrease in risk of tuberculosis infection despite increase in tuberculosis among young adults in urban Vietnam. Int $\mathrm{J}$ Tuberc Lung Dis 2010; 14: 289-95.

[9] Shrestha KB, Malla $\mathrm{P}$, Jha KK, et al. First national tuberculin survey in Nepal. Int J Tuberc Lung Dis 2008; 12: 909-15.

[10] Styblo K. The Relationship between the risk of tuberculous infection and the risk o developing infectious tuberculosis. Bull Int Union Tuberc Lung Dis 1985; 60: 117-9.

[11] Van Leth F, Van der Werf MJ, Borgdorff MW. Prevalence of tuberculous infection and incidence of tuberculosis: a reassessment of the Styblo rule. Bull World Health Organ 2008; 86: 20-6. 
[12] Borgdorff MW. New measurable indicator for tuberculosis case detection. Emerging Infect Dis 2004; 10: 1523-8.

[13] United Nations Millenium Development Goals. Available at: http://www.un.org/millenniumgoals

[14] WHO Global Task Force on TB Impact Measurement. Available at: http://www.who.int/tb/advisory_bodies/impact_measurement_ta skforce/en/

[15] Van der Werf MJ, Borgdorff MW. How to measure the prevalence of tuberculosis in a population. Trop Med Int Health 2007; 12: 47584.

[16] Tuberculosis prevalence surveys: a handbook. $2^{\text {nd }}$ ed. Geneva, Switzerland: WHO 2011

[17] Sebhatu M, Kiflom B, Seyoum M, et al. Determining the burden of tuberculosis in Eritrea: a new approach. Bull World Health Organ 2007; 85: 593-9.

[18] Lönnroth K, Holtz TH, Cobelens F, et al. Inclusion of information on risk factors, socio-economic status and health seeking in a tuberculosis prevalence survey. Int J Tuberc Lung Dis 2009; 13: $171-6$

[19] Hoa NB, Tiemersma EW, Sy DN, et al. Health-seeking behaviour among adults with prolonged cough in Vietnam. Trop Med Int Health 2011; 16: 1260-7.

[20] Van Leth F, Guilatco RS, Hossain S, et al. Measuring socioeconomic data in tuberculosis prevalence surveys. Int $\mathrm{J}$ Tuberc Lung Dis 2011; 15 Suppl 2: S58-63.

[21] Hoa NB, Tiemersma EW, Sy DN, et al. Household expenditure and tuberculosis prevalence in VietNam: prediction by a set of household indicators. Int J Tuberc Lung Dis 2011; 15: 32-7.

[22] Hossain S, Quaiyum MA, Zaman K, et al. Socio Economic Position in TB Prevalence and Access to Services: Results from a Population Prevalence Survey and a Facility-Based Survey in Bangladesh. PLoS ONE 2012; 7:e44980.

[23] Hoa NB, Cobelens FGJ, Sy DN, Nhung NV, Borgdorff MW, Tiemersma EW. Yield of interview screening and chest X-ray abnormalities in a tuberculosis prevalence survey. Int $\mathrm{J}$ Tuberc Lung Dis 2012; 16: 762-7.

[24] Banu S, Hossain S, Uddin MKM, et al. Comparison of Macroscopic and Microscopic Assessment of Specimens Collected for the Diagnosis of Tuberculosis. Open Infect Dis J 2012; 6: 1-4.
[25] Begg CB, Greenes RA. Assessment of diagnostic tests when disease verification is subject to selection bias. Biometrics 1983; 39: 207-215.

[26] Zaman K, Hossain S, Banu S, et al. Prevalence of smear-positive tuberculosis in persons aged $\geq 15$ years in Bangladesh: results from a national survey, 2007-2009. Epidemiol Infect 2012; 140: 101827.

[27] Hoa NB, Sy DN, Nhung NV, Tiemersma EW, Borgdorff MW, Cobelens FGJ. National survey of tuberculosis prevalence in Viet Nam. Bull World Health Organ 2010; 88: 273-80.

[28] Global Tuberculosis Control 2011 (WHO/HMT/TB/2011.16) Geneva, Switzerland: World Health Organization; 2011.

[29] Guidelines for surveillance of drug resitance in tuberculosis (WHO/TB/2003.320). Geneva: World Health Organization 2003.

[30] Chonde TM, Basra D, Mfinanga SGM, et al. National antituberculosis drug resistance study in Tanzania. Int $\mathbf{J}$ Tuberc Lung Dis 2010; 14: 967-92.

[31] Chonde TM, Doulla B, Van Leth F, et al. Implementation of a national anti-tuberculosis drug resistance survey in Tanzania. BMC Public Health 2008; 8: 427.

[32] Rigouts L, Hoza AS, De Rijk P, et al. Evaluation of the Genotype ${ }^{\circledR}$ MTBDRplus assay as a tool for drug resistance surveys. Int J Tuberc Lung Dis 2011; 15: 959-65.

[33] Zignol M, Van Gemert W, Falzon D, et al. Surveillance of antituberculosis drug resistance in the world: an updated analysis, 2007-2010. Bull World Health Organ 2012; 90: 111-9D.

[34.] Huong NT, Lan NTN, Cobelens FGJ, et al. Antituberculosis drug resistance in the south of Vietnam: prevalence and trends. J Infect Dis 2006; 194: 1226-32.

[35] Buu TN, Van Soolingen D, Huyen MNT, et al. Increased transmission of Mycobacterium tuberculosis Beijing genotype strains associated with resistance to streptomycin: a populationbased study. PLoS ONE 2012; 7:e42323.

[36] Hedt BL, Van Leth F, Zignol M, et al. Multidrug resistance among new tuberculosis cases: detecting local variation through lot quality-assurance sampling. Epidemiology 2012; 23: 293-300.

(C) van Leth and Klinkenberg; Licensee Bentham Open.

This is an open access article licensed under the terms of the Creative Commons Attribution Non-Commercial License (http: //creativecommons.org/licenses/by$\mathrm{nc} / 3.0 /$ ) which permits unrestricted, non-commercial use, distribution and reproduction in any medium, provided the work is properly cited. 